\title{
Production of Pure Nano-Iron by using ball milling machine, chemical batch reactor and K-M micro reactor
}

\author{
Mohamed Ahmed AbdelKawy ${ }^{1, *}$, Ahmed H. El-Shazly ${ }^{1}$, Yehia El Shazly ${ }^{2}$ \\ ${ }^{1}$ Chemical and Petrochemical Engineering Dept., Egypt-Japan University of Science and Technology (E-JUST), New Borg ELArab city, \\ Alexandria, Egypt \\ ${ }^{2}$ Chemical Engineering Dept., Faculty of Engineering, Alexandria University, Alexandria, Egypt \\ Email address: \\ Mohammed.hammad@ejust.edu.eg (M. A. AbdelKawy), elshazly_a@yahoo.com (A. H. El-Shazly), \\ yehiaelshazly@hotmail.com (Y. E. Shazly)
}

\section{To cite this article:}

Mohamed Ahmed AbdelKawy, Ahmed H. El-Shazly, Yehia El Shazly. Production of Pure Nano-Iron by Using Ball Milling Machine, Chemical Batch Reactor and K-M Micro Reactor. American Journal of Applied Chemistry. Special Issue: Nano-technology for Environmental Aspects. Vol. 3, No. 3-1, 2015, pp. 8-12. doi: 10.11648/j.ajac.s.2015030301.12

\begin{abstract}
In this study, pure nano iron was produced by the top to down and bottom up techniques. top to down including production of nano iron by using ball mill machine and bottom up including two methods batch and K-M micro reactor. Different techniques were used for investigation and characterization of the produced nano iron particles such as UV-Vis, PSD, SEM, TEM and XRD. The produced Nano particle using micro mixer showed better characteristics than those produced using batch reactor and ball mill in many aspects such as homogeneity of the produced particles, particle size distribution and size. The results showed that $10 \mathrm{~nm}$ core diameter were obtained using Micro mixer as compared to $80 \mathrm{~nm}$ and $40 \mathrm{~nm}$ core diameter using batch reactor and ball mill respectively.
\end{abstract}

Keywords: Nano Iron, Micro Reactor, Batch Reactor, Ball Mill

\section{Introduction}

Nanotechnology is the engineering and art of manipulating matter at the Nano scale $(1-100 \mathrm{~nm})$ [1]. For environmental applications, nanotechnology offers the potential of novel functional materials, processes and devices with unique activity toward recalcitrant contaminants, enhanced mobility in environmental media and desired application flexibility [1-3]. Many Nano-based environmental technologies (e.g., sensors, sorbents, and reactants) are under very active research and development, and are expected to emerge as the next generation environmental technologies to improve or replace various conventional environmental technologies in the near future [1].

Iron nanoparticles technology represents perhaps one of the first generation Nano scale environmental technologies [4]. Over the last few years, various synthetic methods have been developed to produce iron nanoparticles [5], modify the nanoparticles surface properties [5], and enhance the efficiency for field delivery and reactions. Extensive laboratory studies have demonstrated that Nano scale iron particles are effective for the transformation of a wide array of common environmental contaminants such as chlorinated organic solvents, organo chlorine pesticides, PCBs, organic dyes, various inorganic compounds and metal ions such as $\mathrm{As}(\mathrm{III}), \mathrm{Pb}(\mathrm{II}), \mathrm{Cu}(\mathrm{II}), \mathrm{Ni}(\mathrm{II})$ and $\mathrm{Cr}(\mathrm{VI})$. Several field tests have demonstrated the promising prospective for in situ remediation [6-8] .Many research papers on applications of iron nanoparticles have been published over the last few years. While several types of iron nanoparticles are available on the market, information on the nanoparticles synthesis and properties is still limited in peer reviewed journals. Fundamental information on characterization methods has not been well documented. Quality control and insurance is rapidly becoming a major issue as nanoparticles are being used in more and more projects. The main objective of this work is to compare between the main methods used for production of Nano iron particles such as ball milling machine and using chemical processes either in batch reactor or in K-M micro mixer and to optimize and find out the main parameters controlling the characteristics of produced Nano iron, to produce Nano iron of higher value. 


\section{Experimental}

\subsection{Top to Down Method}

\subsubsection{Using Ball Mill}

In this method, commercial pure iron was milled by using planetary ball milling machine as shown in Fig.1, this mill consists of 4 jars, two of the them fill with iron mixed with ethanol to prevent oxidation of iron and other fill with sand for stability under high centrifugal force, two forces were affected for decreasing the size of this pure iron, one high impact force due action of balls and friction force due to centrifugal force, two different ball size using for milling 30 $\mathrm{mm}$ and $10 \mathrm{~mm}$. After milling time ranging from 25 to $35 \mathrm{hr}$, produced iron was dried over hot plate and then characterized using different techniques.

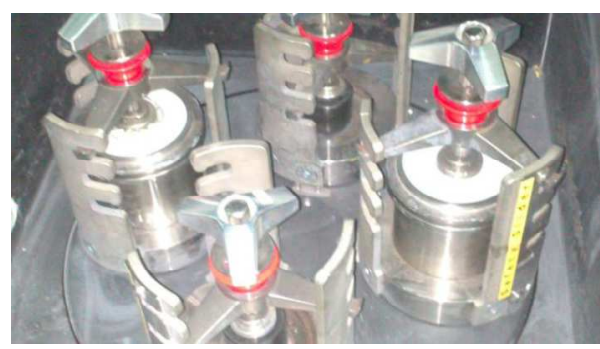

Figure 1. Planetary Ball Mills-PM 400

\subsection{Bottom up Process}

\subsubsection{Mixing in Batch Reactor}

As shown in Fig.2, batch reactor of $500 \mathrm{ml}$ size with three necks was used to prepare zero valent iron (nZVI). A magnetic stirr was used for solution agitation and magnetic separator was used to separate the produced (nZVI). The main reaction involved is:

$$
\begin{gathered}
2 \mathrm{FeCl}_{3}+6 \mathrm{NaBH}_{4}+18 \mathrm{H}_{2} \mathrm{O}=2 \mathrm{Fe} 0+6 \mathrm{NaCl}+6 \mathrm{~B}(\mathrm{OH})_{3} \\
+21 \mathrm{H}_{2}
\end{gathered}
$$

(nZVI) was synthesized by using batch rector; $4.83 \mathrm{~g}$ $\mathrm{FeCl}_{3} \cdot 6 \mathrm{H}_{2} \mathrm{O}$ was dissolved in a $4 / 1(\mathrm{v} / \mathrm{v})$ ethanol/water mixtrue $(216 \mathrm{ml}$ ethanol $+54 \mathrm{ml}$ deionized water $)$ and stirred well by using magnetic stirrer[2] .

On the other hand, $0.614 \mathrm{M}$ sodium borohydride solution was prepared by dissolving $6.30 \mathrm{~g} \mathrm{NaBH}_{4}$ in $100 \mathrm{ml}$ of deionized water [2]. The borohydride solution was added drop wise (1drop per 2 seconds) into iron chloride solution.

After the first drop of sodium borohydride solution, black solid particles immediately appeared and then the remaining sodium borohydride is added completely to accomplish the reduction reaction. The mixture was left for another 10 minutes of stirring after adding the whole borohydride solution [2]. Magnetic separator was used to separate the black iron nanoparticles from the liquid phase. The solid particles were washed three times with $25 \mathrm{ml}$ portions of absolute ethanol to remove all of the water and prevent the rapid oxidation of (nZVI) [2]. The synthesized nanoparticles were finally dried in vacuum oven at $70{ }^{\circ} \mathrm{C}$ overnight.

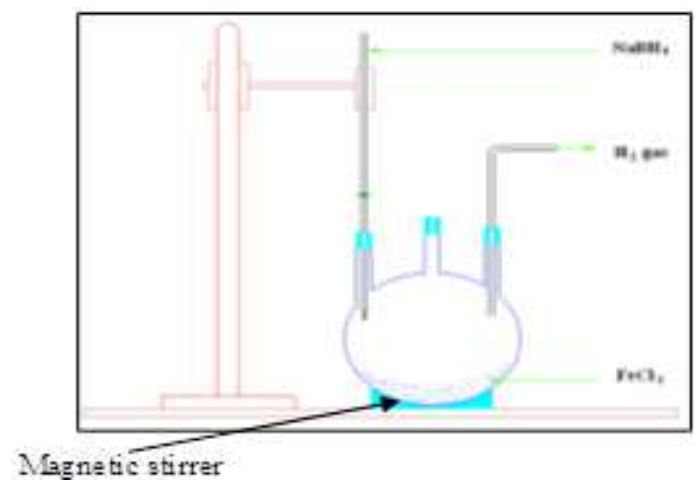

Figure.2. Experimental setup of batch reactor

\subsubsection{Mixing by $K$-M Mixer}

As shown in Fig.3, K-M micro mixer was used for the production of (nZVI) using the same conditions described in section 2.2.1. the details description of K-M micro mixer can be reviewed in Ref.[3] $\mathrm{FeCl} 3.6 \mathrm{H} 2 \mathrm{O}$ and $\mathrm{NaBH} 4$ were injected to the K-M micro mixer using a syringe pump with a constant flow rate of $373 \mathrm{ml} / \mathrm{h}$. The produced solid Nano particles were collected in $100 \mathrm{ml}$ container and filter in the presence of magnetic separator then washed three times with $25 \mathrm{ml}$ portions of absolute ethanol to remove all of the water and prevent the rapid oxidation of (nZVI) nanoparticles. The synthesized nanoparticles were finally dried in vacuum oven at $70{ }^{\circ} \mathrm{C}$ overnight.

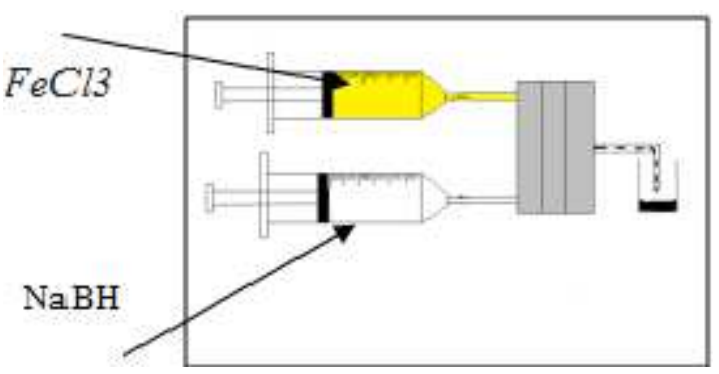

Figure 3. Experimental setup of $K-M$ mixer

\section{Characterisation of (nZVI) Produced}

The produced (nZVI) in ethanol was immediately analyzed by a UV spectrometer (shimadzu, Ltd., Multispec-1500), Particles Size Distribution analyzer (PSD-Melvern-2000) and Transmutation Electron Microscope (TEM-Jeol-1010). Solid (nZVI) was analyzed by using Scan Electron Microscope (SEM-Jeol-2000).

\section{Results and Discussion}

As shown in Figs.4.a, b and c, the experiment was carried out in the range from wavelength of 200 to $500 \mathrm{~nm}$. The 
results show that the particles produced from all techniques has an absorbance peak within the range from 210 to 240 which confirms the nano range of particles size [2]

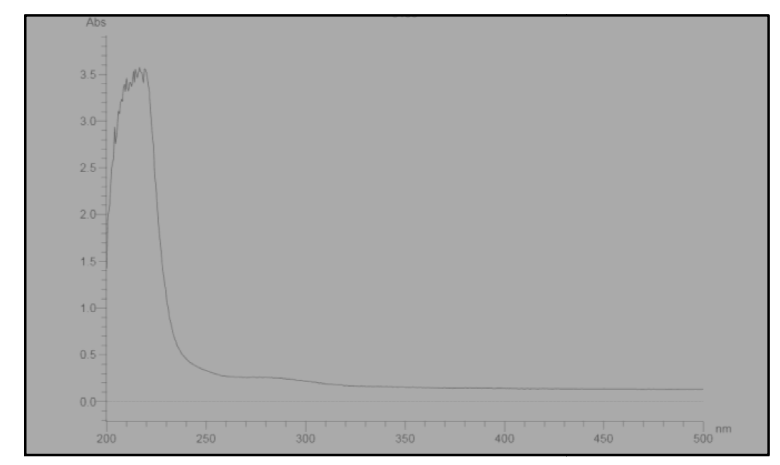

(a)

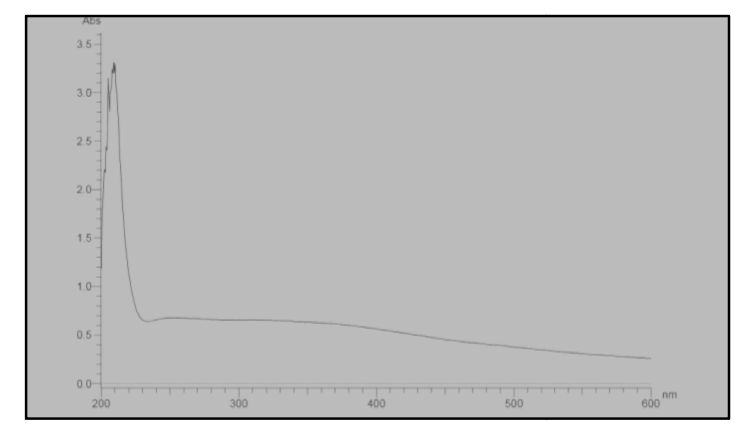

(b)

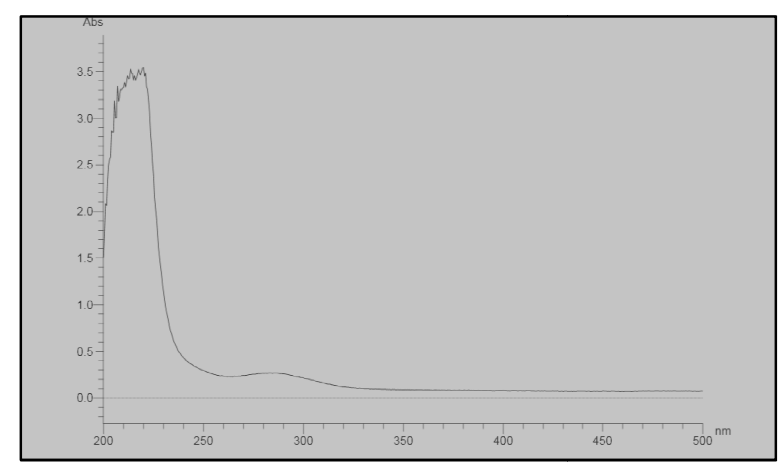

(c)

Figure 4. UV-Vis of Nano iron produced on a-ball milling, b-batch reactor, c-K-M Micro mixer

Figs.5a, b and c show the particle size distribution of iron particles before milling, after 25 and $35 \mathrm{hr}$ of milling respectively. The results show that milling is effective in reducing the particle size with time, as the size was reduced from 150 to $1.5 \mu \mathrm{m}$ and then to $0.15 \mu \mathrm{m}$ after milling for 25 and $35 \mathrm{hr}$ respectively. On the other hand as shown in Figs.5.d and e, batch reactor and K-M micro mixer were shown to be effective in producing the (nZVI) in shorter time of $40 \mathrm{~min}$ and $5 \mathrm{~min}$ respectively. The average particles size produced was $11 \mu \mathrm{m}$ and $0.7 \mu \mathrm{m}$ for batch and $\mathrm{k}-\mathrm{m}$ micro mixer respectively. Overall the particle size distribution analysis shows that in spite of milling produced fine particles in the range of $0.150 \mu \mathrm{m}(150 \mathrm{~nm})$

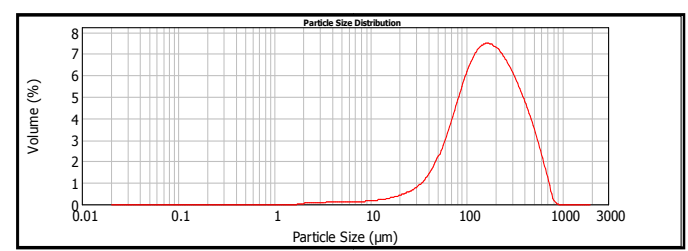

(a)

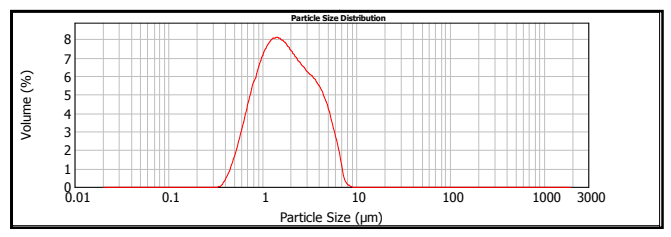

(b)

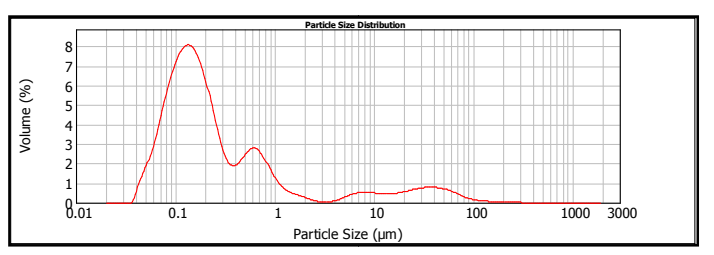

(c)
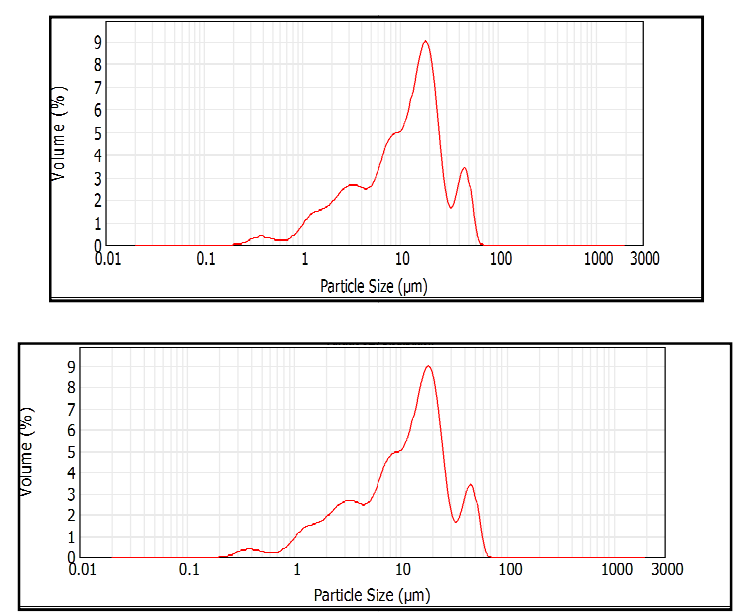

(d)
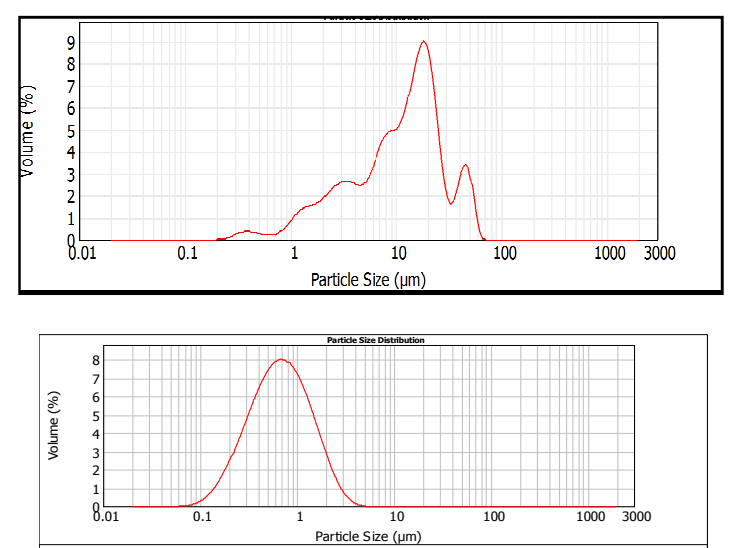

(e)

Figure 5. Particle size analyzer a-before milling, b-after $25 \mathrm{hr}$. of milling, $c$-after $35 \mathrm{hr}$. of milling-after mixing in batch reactor, e-after mixing in $K-M$ Micro mixer. 
$\mathrm{K}-\mathrm{M}$ micro mixer was proved to produce a homogenous product of (nZVI) at very short time which considers an economic advantage of the process. Also, as shown in Fig.6.a, $\mathrm{b}$, c and d, SEM images show much more homogenous particle size produced for $\mathrm{K}-\mathrm{M}$ micro mixer as shown in Fig.6.d.

On the other side, as shown in Fig.7a,b and c,TEM images show very clear images of Nano iron produced using different techniques, from these images the sizes of Nano iron produced were 40,80 and $10 \mathrm{~nm}$ for milling, batch and K-m micro mixer respectively

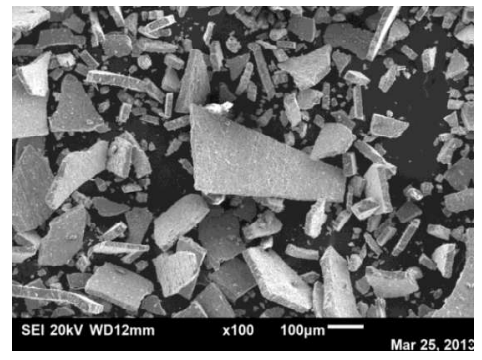

(a)

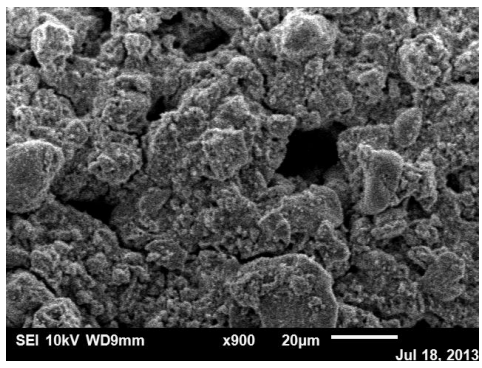

(b)

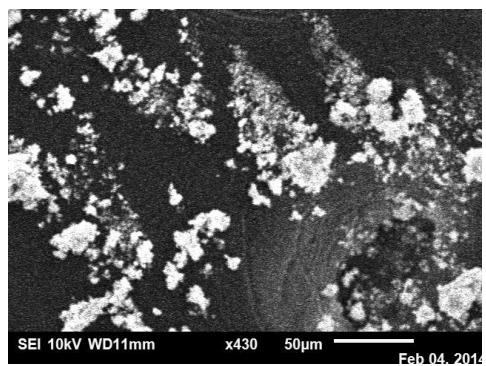

(c)

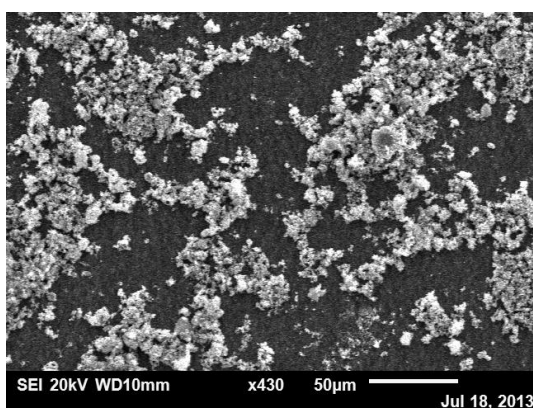

(d)

Figure 6. SEM of Nano iron a-before milling, b-after $35 \mathrm{hr}$. of milling c-batch reactor, $d-K-M$ micro mixer

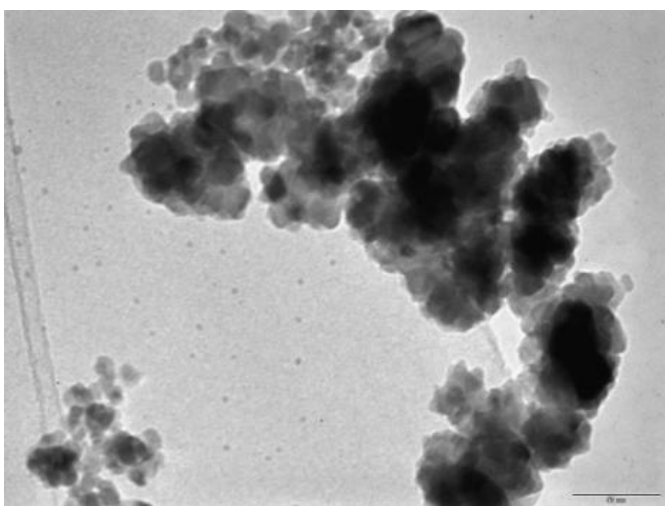

(a)

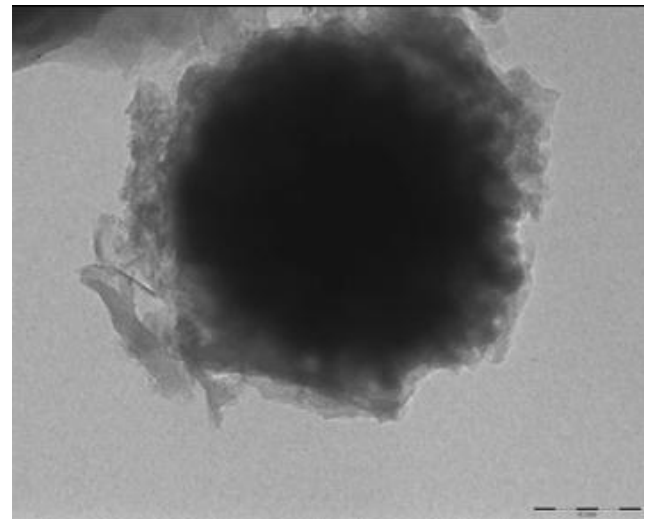

(b)

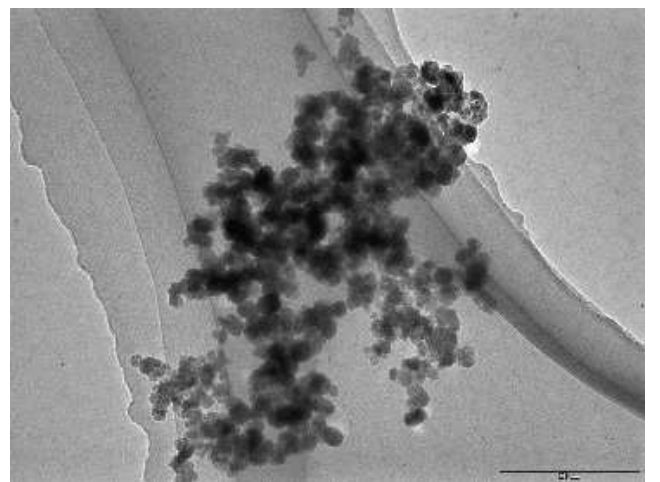

(c)

Figure 7. TEM images of Nano iron produced $a$-ball mill, $b$ - batch reactor [2], c- K-M Micro mixer

These results contradicted with the particle size distribution results in respect to the size obtained using different techniques. However, it is worthy to mention that the particle obtained from milling and batch reactor has agglomerates while these obtained using K-M micro mixer are homogenous and well dispersed.

The powder XRD pattern of nano iron samples under ambient conditions shown in Fig.8. The characteristic broad peak at $2 \theta$ of $45^{\circ}$ indicates that the zero valent iron is predominantly present in samples produced by K-M Micro reactor and batch reactor as shown in Fig.7-b but at milling conditions, peaks at $35^{\circ}, 54^{\circ}$, and $33^{\circ}$ that is due to oxidation of iron after milling as shown in Fig.7-a. 


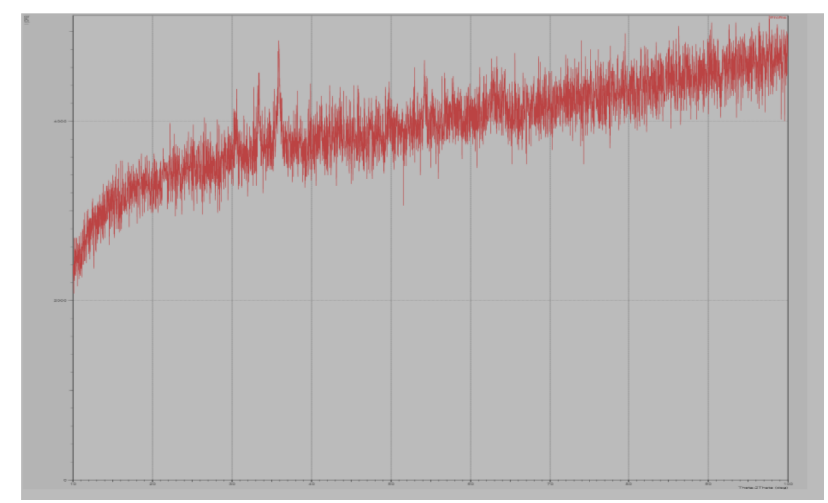

(a)

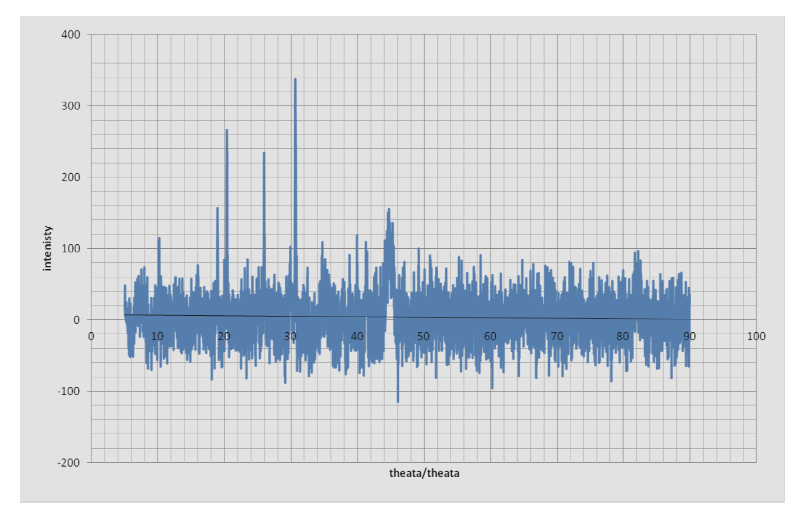

(b)

Figure 8. XRD of Nano iron produced, a-ball milling, $b$ - K-M Micro, batch reactor

\section{Conclusion}

Three techniques were compared for the production of (nZVI) namely milling, batch reactor and k-m micro mixer. Different techniques such as UV-Vis, PSD, SEM, TEM and XRD were used for the charactrisations of the produced (nZVI). The results showed that (nZVI) can be produced by using three techniques while milling has the advantage of producing lower size, however it has many drawbacks such as time, power and partial oxidation of the produced(nZVI). $\mathrm{K}-\mathrm{m}$ micro mixer was proved to produce (nZVI) with higher homogeneity and well dispersed particles in addition to the very short time required for the reaction, which improves the economy of the produced (nZVI).

\section{Acknowledgement}

The first author is supported by a scholarship from the Mission Department, Ministry of Higher Education of the Government of Egypt, which is gratefully acknowledged. Our sincere thanks are extended to Egypt -Japan University of science and technology (E-JUST) for guidance and support. Also we would like to acknowledge Prof.Kazuhiro Mae (Environmental Process Engineering Lab.,) and Dr. Satoshi Watanabe (Surface Control Lab.,), Chemical Engineering Department, Graduate School of Engineering, Kyoto University for their valuable advices, guidance and providing the K-M micro mixer.

\section{References}

[1] Y.Sun, X.Li , J. Cao, W.Zhang, H. Wang 'Characterization of zero-valent iron nanoparticles' Advances in Colloid and Interface Science,2006, 120 ,47-56.

[2] R. Yuvakkumar, , V. Elango, V. Rajendran, N. Kannan ' Preparation and characterization of zero valent iron nano particles' Digest Journal of Nanomaterials and Biostructures, 2011,6, 4, 1771-1776.

[3] H. Nagasawa, N. Aoki, K. Mae 'Design of a new micromixer for instant mixing based on the collision of micro segments' Chem.Eng.Technol,2005,28,3,30-35 .

[4] A. R. Rahmani, H.R. Ghaffari, M.T. Samadi 'A Comparative study on arsenic (III) removal from Aqueous Solution using nano and micro sized zero valent iron ' Iran. J. Environ. Health. Sci. Eng., 2011, 8, 2, 175-180.

[5] X. Li, D. Elliott, W. Zhang ' Zero-Valent Iron Nanoparticles for Abatement of Environmental Pollutants' Materials and Engineering Aspects Critical Reviews in Solid State and Materials Sciences, 2006,31,111-122

[6] W. Yaacob, N. Kamaruzaman, A.Samsudin 'Development of Nano-Zero Valent Iron for the Remediation of Contaminated Water' Chemical Engineering Transactions, 2012, 28,100-107.

[7] A. Akbari, , F. Mohamadzadeh 'New Method of Synthesis of Stable Zero Valent Iron Nanoparticles (Nzvi) by Chelating Agent Diethylene Triamine Penta Acetic Acid (DTPA) and Removal of Radioactive Uranium From Ground Water by using Iron Nanoparticles', JNS, 2012,2 - 175-181.

[8] W. Zhang 'Nanoscale iron particles for environmental remediation: An overview 'Review Journal of Nanoparticles Research, 2002, 5, 323-332 